\title{
Melt Electrowriting of Electroactive Poly(vinylidene difluoride) Fibers
}

\author{
Sammy Florczak ${ }^{1,2}$ Thomas Lorson ${ }^{3}$, Tian Zheng ${ }^{4,5}$, Miroslav Mrlik ${ }^{3,6}$, \\ Dietmar W. Hutmacher ${ }^{2}$, Michael J. Higgins ${ }^{4}$, Robert Luxenhofer ${ }^{3 *}$, Paul \\ D. Dalton ${ }^{1 *}$
}

${ }^{1}$ Functional Materials in Medicine and Dentistry and Bavarian Polymer Institute, University Clinic Würzburg, Würzburg, Germany;

${ }^{2}$ Institute for Health and Biomedical Innovation, Queensland University of Technology, Brisbane, Australia;

${ }^{3}$ Polymer Functional Materials, Chair for Advanced Materials Synthesis, Department of Chemistry and Pharmacy, Julius-Maximilians-University Würzburg, Würzburg, Germany;

${ }^{4}$ ARC Centre of Excellence for Electromaterials Science, Intelligent Polymer Research Institute, University of Wollongong, Wollongong, Australia;

${ }^{5}$ Materials Characterisation and Fabrication Platform, University of Melbourne, Melbourne, Australia;

${ }^{6}$ Centre of Polymer Systems, University Institute, Tomas Bata University in Zlin, Trida T. Bati 5678, 760 01, Zlin, Czech Republic.

Correspondence to:

Prof. Dr. Robert Luxenhofer: robert.luxenhofer@uni-wuerzburg.de

Prof. Dr. Paul Dalton: paul.dalton@fmz.uni-wuerzburg.de

This is the author manuscript accepted for publication and has undergone full peer review but has not been through the copyediting, typesetting, pagination and proofreading process, which may lead to differences between this version and the Version of Record. Please cite this article as doi: $10.1002 /$ pi.5759

This article is protected by copyright. All rights reserved. 


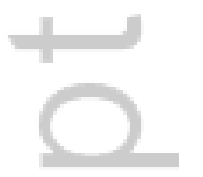




\begin{abstract}
Poly(vinylidene difluoride) (PVDF) has piezoelectric properties suitable for numerous applications such as flexible electronics, sensing and biomedical materials. In this study, individual fibers with diameters ranging from 17-55 $\mu \mathrm{m}$ were processed using melt electrowriting (MEW). Electroactive PVDF fibers can be fabricated via MEW, while the polymer could remain molten for up to ten hours without noticeable changes in the resulting fiber diameter. MEW processing parameters for PVDF were investigated, including applied voltage, pressure and temperature. A rapid fiber characterization methodology for MEW that automatically determines the fiber diameters from camera images taken of microscope slides was developed and validated. The outputs from this approach followed previous MEW processing trends already identified with different polymers, although overestimation of the $<25$ micron fiber diameters was observed. The transformation of the PVDF crystalline phase to the electroactive $\beta$-phase - without poling - was confirmed using piezo-force microscopy and revealed that the PVDF fibers possess piezoelectric responses showing $\mathrm{d}_{33} \sim 19$ $\mathrm{pm} / \mathrm{V}$.
\end{abstract}

Keywords: 3D printing; piezoelectric; piezoresponse force microscopy; electrohydrodynamic; melt electrospinning writing; 


\section{Introduction}

Additive manufacturing (AM), also termed 3D printing, comprises of numerous fabrication technologies that use layer-by-layer principles to fabricate an object (1). Nozzle-based direct-writing is a significant and important class within AM, and is commonly performed with polymer melts $(2)$, shear-thinning hydrogels $(3,4)$ or colloidal ink formulations (5). For viscous fluids including polymer melts, there are lower dimensional limits to the extruded diameter, due to a physical phenomenon termed the Barus effect also known as die/extrudate swell (6). Recently, there is an increasing number of publications that circumvent the effects of die swell through the application of a high voltage as the material is being extruded at low flow rates (7). The enhanced, electrostatic drawing of the fluid as it passes out of the nozzle is situated within the broader area of electrohydrodynamic jetting (8), where continuous filaments or non-continuous droplets are precisely placed onto a collector. In this study, we employ melt electrowriting (MEW) (7), which is distinct from electrospinning (9), in that electrical instabilities are suppressed to precisely place fibers but sufficient to maintain a fluid column from breaking at low flow rates (10). When the collector speed for MEW matches the jet speed - widely termed the critical translation speed (CTS) this fluid column can be direct-written as a linear fiber $(11,12)$.

Electrospinning is one field of research that dominates recent fiber-forming research literature, due to simplicity, low-cost and ability to produce small diameter fibers (13). The promotion of electrical instabilities within a charged fluid jet, and a long jet path to aid in solvent evaporation readily result in nano- and ultra-fine fibers. A 
major challenge with applying AM principles to electrospinning is that the fiber placement cannot be controlled well enough for precise layer-by-layer fabrication. Polymer melts can also be electrospun (14) but can be effectively direct-written due to their typically higher viscosity which results in a stable liquid/molten jet and rapid solidification (15). Electrospun fibers from melts tend to have (but not always) larger diameters than those obtained from a polymer solution (16), but significantly smaller diameters than most direct-written, extruded polymer melts (17). Such well-placed micron-diameter fibers provide mechanical and biological properties not seen with solution electrospun meshes $(18,19)$ and bridge the gap in a size regime that is relevant for numerous applications.

To date, only six polymers, namely poly( $\mu$-caprolactone) (PCL), poly(propylene) (PP), poly(2-ethyl-2-oxazoline), poly(hydroxymethyl glycolide-co- $\mu$-caprolactone) (PHMGCL), poly(urea-siloxane) and poly(L-lactide-co- $\mu$-caprolactone-co-acryloyl carbonate), have been shown to be processable via MEW (20-25). To successfully establish MEW for different polymers, it is typically required that a preliminary investigation is undertaken to determine the influence of printing parameters on the fiber diameter and placement. Such an analysis establishes a basis for understanding the range of parameters over which the polymer can be processed, hence revealing what fiber diameters and 3D printing resolution can be achieved. Many instrument parameters have an influence on the MEW jet (Figure 1), and consequently the resultant fibers and scaffolds (11), making the establishment of appropriate printing conditions a multi-parameter challenge. 
In this study, poly(vinylidene fluoride) (PVDF) was chosen as a candidate for processing via MEW. It is a well-known polymer with electroactive properties and has been previously fabricated as a fiber mat by solution electrospinning (26), to provide a system with enhanced electroactive properties (27). The electroactivity of PVDF is mainly based on the transformation of the non-electrically active $\alpha$-crystalline phase to electroactive $\beta$-crystalline phase. It was reported that such transformation can be performed using particle addition (28), electrospinning (29), or poling (30). Since the MEW technique is similar to solution electrospinning it is expected that this technique can be applicable for the fabrication of the fibers with enhanced $\beta$-crystalline phase and thus with promising piezoelectric activity.

Previous reports have demonstrated that parameters such as the flow rate of molten polymer from the nozzle, the applied potential difference between the nozzle and collector, the collector speed and the collector distance (the distance between the nozzle and the collector) defines the morphological properties of a directly-written fibers (11) (Figure 1). Given this multi-parametric nature of MEW, the ability to measure a large number of samples become essential to optimize the parameters. The primary method for measuring fiber diameters is scanning electron microscopy (SEM), however from a practical perspective, it is both time-consuming and an expensive method to quantify the diameter of fibers when so many parameters require investigation. This is further exacerbated when fibers are printed at least in triplicate, and all samples require inclusion within the data set.

\section{INSERT FIGURE 1 NEAR HERE}


Finally, the elevated temperature necessary for MEW and the prolonged residence time in the electrically-heated chamber and nozzle, the thermal degradation of the printed polymer is another factor that requires consideration (23). With such a large parameter set relevant for MEW (Figure 1), it becomes clear that methods for processing a significant quantity of data become necessary. For this purpose, the use of SEM has limited efficacy in the high-throughput analysis of MEW fibers.

Previously, software specifically designed for the analysis of micrographs containing fibers have proven to be highly useful for the bulk analysis of solution electrospun scaffolds, especially for non-woven mats which are intrinsically difficult to measure due to the stochastic nature of the deposited fiber. However, since the MEW fibers often have micron-scale diameters, we can explore the combination of optical microscopy with such experimental design, to generate a powerful tool in understanding the different instrument parameters that affect MEW processing.

In this study, we establish MEW with PVDF and introduce a protocol for using Image J and the plug-in DiameterJ for extracting information on the fiber diameters using optical methods. The development of automated measurement of fiber diameter is advantageous for the characterization of hundreds or even thousands of samples in a relatively short period of time with minimal input from the user. The automated measurement process prevents user bias as the entirety of the sample, including outliers such as long beading (11), are included in the measurement, thus painting a more accurate and complete picture of direct-writing conditions. To further analyze the 
properties of the PVDF fibers, electroactivity measurements on the MEW fibers were performed. The capacity to produce fine $3 \mathrm{D}$ printed piezoelectric structures through MEW may pave the way for novel applications in compact sensing or energy harvesting technologies $(29,31)$.

\section{Materials and Methods}

\section{Materials}

Poly(vinylidene difluoride) (Piezotech Kynar RC10.287) was received as a generous gift from Piezotech (Pierre-Benite, France). The white, odorless powder was used as received.

\section{Differential scanning calorimetry}

Differential scanning calorimetry (DSC) was performed on a DSC/TG STA409 PC (NETZSCH, Selb, Germany) under $\mathrm{N}_{2}$-atmosphere $(20.0 \mathrm{~mL} / \mathrm{min})$. The samples were placed in aluminium oxide crucibles (NETZSCH, Selb, Germany), heated to $220{ }^{\circ} \mathrm{C}(5 \mathrm{~K} / \mathrm{min})$ and subsequently cooled to $30{ }^{\circ} \mathrm{C}(5 \mathrm{~K} / \mathrm{min})$ before heating to $220{ }^{\circ} \mathrm{C}$ for a second time $(5 \mathrm{~K} / \mathrm{min})$. To evaluate the spectra NETZSCH Proteus - Thermal Analysis - V.4.2.1 software was used.

\section{Gel Permeation Chromatography}

Gel permeation chromatography (GPC) was carried out on a Polymer Standard Service SECcurity (PSS, Mainz, Germany) system (pump mod. 1260 infinity, MDS RI detector mod. 1260 infinity (Agilent Technologies, Santa Clara, California, USA), precolumn PSS GRAM $10 \mu \mathrm{m}(50 \times 8 \mathrm{~mm})$, PSS GRAM $30 \AA 10 \mu \mathrm{m}(300 \times 8 \mathrm{~mm})$ 
and PSS GRAM $1000 \AA 10 \mu \mathrm{m}(300 \mathrm{x} 8 \mathrm{~mm}))$ at $313 \mathrm{~K}$. The mobile phase (dimethylformamide) was supplemented with $1 \mathrm{~g} / \mathrm{L} \mathrm{LiBr}$ and the flow rate was adjusted to $1 \mathrm{~mL} / \mathrm{min}$. Prior to each measurement, the samples were filtered through a $0.2 \mu \mathrm{m}$ Teflon filter (Thermo Scientific) to remove particles, if present. The system was calibrated against PEG standards with molar masses ranging from $106 \mathrm{~g} / \mathrm{mol}$ to $100 \mathrm{~kg} / \mathrm{mol}$. Data were processed using WinGPC Unichrom V.8.20 Build 5350 software.

\section{Thermogravimetric analysis}

Thermogravimetric analysis of the polymers was performed on a TG 209F1 IRIS (NETZSCH, Selb, Germany). The samples $(5-10 \mathrm{mg})$ were added to aluminum oxide crucibles (NETZSCH, Selb, Germany) and heated under synthetic air from $30^{\circ} \mathrm{C}$ to $180{ }^{\circ} \mathrm{C}$ or $190{ }^{\circ} \mathrm{C}$, with a heating rate of $10 \mathrm{~K} / \mathrm{min}$. The temperature was kept constant for $5 \mathrm{~h}$ while detecting the mass loss. The corresponding NETZSCH Proteus - Thermal Analysis - V.5.2.1 software was used to evaluate the obtained spectra.

\section{Rheology}

Rheology experiments were performed using a Physica MCR 301 (Anton Paar, Graz, Austria) equipped with a Toolmaster ${ }^{T M}$ measuring cell and measuring system as well as with a Peltier element. A plate-plate setup was utilized equipped with an upper $25 \mathrm{~mm}$ plate. To ensure a preferably constant temperature a protective cover was used. Data was processed using the Physica RheoPlus V.3.40 software (Anton Paar, Graz, Austria).

MEW Processing

This article is protected by copyright. All rights reserved. 
MEW of PVDF was performed with a custom-built device which operated by pneumatically driving the melt with $\mathrm{N}_{2}$ through a 26-gauge electrified stainless-steel nozzle screwed onto a $3 \mathrm{~mL}$ temperature controlled glass syringe (32). The heating of the syringe/melt was accomplished through the use of two resistive heating elements positioned at the tip and body of the syringe, with both being controlled through individual thermocouples and a two-channel proportional-integral-derivative (PID) controller. These were set to a temperature of $190 \pm 2{ }^{\circ} \mathrm{C}$ as reported by each thermocouple and re-verified with an external temperature probe (Voltcraft K202 Datalogger Thermometer) which measured $190 \pm 1^{\circ} \mathrm{C}$ at the nozzle heater, $190 \pm 1{ }^{\circ} \mathrm{C}$ at the reservoir heater, and $189 \pm 2{ }^{\circ} \mathrm{C}$ within the fluid, with uncertainties arising from observed fluctuations in the measurement depending on the position the thermocouple. The flat-tipped nozzle was made by cutting and grinding the needle to a length of $5.0 \pm$ $0.2 \mathrm{~mm}$. A potential difference of $+2.70 \pm 0.08 \mathrm{kV}$ was applied between the nozzle and the grounded collector plate as measured by an external high-voltage probe (TESTEC HVP40) and multi-meter. The electrowriting was maintained at a working distance of $4.0 \pm 0.5 \mathrm{~mm}$ between the nozzle and collector plate, with $1.0 \pm 0.2 \mathrm{~mm}$ of the nozzle protruding beyond the electrowriting heating head.

Glass microscope slides (VWR, ground edge, cat \# 631-1552) were chosen as a printing substrate in order to facilitate the use of optical microscopy for imaging and to enable easy handling of the structures. The slides were first prepared by rinsing in deionized water and subsequent washing in $100 \%$ ethanol. The slides were then wiped with microfiber tissue and allowed to air dry. Once dry, $4 \mathrm{~mm}$ wide strips of double- 
sided tape were applied to both long edges of one face. The purpose of the double-sided tape was to ensure the scaffold did not lift or detach from the surface during printing or imaging. This approach readily allowed for both analysis of fibers as no region of importance was obstructed, and ensured the printed material would remain stable and well-orientated on the slide during handling. Clean glass syringes (Fortuna Optima $3 \mathrm{~mL}$ Luer Lock) were fully loaded with the polymer powder then compacted using a plunger. This process consistently resulted in an initial volume of $1.0 \pm 0.2 \mathrm{~mL}$ of PVDF melt in the syringe and also reduced the formation of bubbles.

\section{MEW Printing Sequence}

Three different driving pressures were tested per slide for a total of six pressures over two slides - this constituted a single run (Figure 2). For each pressure setting, consecutive fiber triplets within the array were printed with collector speeds ranging from $1800 \mathrm{~mm} / \mathrm{min}$ to $5000 \mathrm{~mm} / \mathrm{min}$ at $400 \mathrm{~mm} / \mathrm{min}$ increments. This arrangement enabled for 54 combinations of parameters to be investigated using only two microscope slides. Between each pressure transition, a stabilization routine was performed, whereby a deposition of fibers was electrowritten off the collector slide for a three-minute duration. This was done to mitigate some of the instability that is commonly seen when transitioning to a different applied pressure (33). To measure temperature dependent changes over time, each set of two slides (corresponding to one parameter sweep) were printed at $\mathrm{t}=0 \mathrm{~h}, 0.5 \mathrm{~h}, 1.0 \mathrm{~h}, 2.0 \mathrm{~h}, 4.0 \mathrm{~h}, 8.0 \mathrm{~h}$ and $10.0 \mathrm{~h}$ of heating, with the molten polymer maintained at a constant printing temperature even when idle. Each time experiment was performed in triplicate. 


\section{INSERT FIGURE 2 NEAR HERE}

\section{Image Processing of Fibers}

The analysis of the PVDF fibers was performed using the image processing tool DiameterJ, a plug-in for the ImageJ/Fiji software (34). DiameterJ is open source nanofiber diameter measurement tool which employs the use of Euclidean distance transforms on a binary segmented image of the fibers in order to determine their centerline and diameter. The tool was initially created for the analysis of solution electrospun fibers imaged through electron microscopy (34) and has been used extensively for fiber analysis applications $(35,36)$. The tool relies on several subpackages such as Segment within the ImageJ/Fiji application to function: conversion from an 8-bit micrograph to a binary image is achieved using a Statistical Region Merging algorithm (34) to define the threshold between the borders of the fibers and the background (Figure 2A-C). Upon noise reduction and computation of fiber diameters, a histogram containing all measured diameters in the image is generated.

\section{SEM Imaging}

SEM imaging of the MEW-processed fibers and scaffolds was performed on a Crossbeam 340 (Carl Zeiss Microscopy GmbH, Germany) with all samples imaged uncoated.

$\beta$-phase content calculation 
As previously published by several research groups $(37,38)$, the $\beta$-phase content can be calculated from the FTIR spectra according to the equation 1:

$$
F(\beta)=\frac{A_{\beta}}{\left(\frac{\kappa_{\beta}}{\kappa_{\alpha}} A_{\alpha}+A_{\beta}\right)}
$$

where, $A_{\alpha}$ and $A_{\beta}$ are values of absorbance corresponding to the wavenumber $762 \mathrm{~cm}^{-1}$ and $840 \mathrm{~cm}^{-1}$, respectively. The $\kappa_{\alpha}$ and $\kappa_{\beta}$ are absorption coefficients for $\alpha$-crystalline phase and $\beta$-crystalline phase, having values $6.110^{4} \mathrm{~cm}^{2} \mathrm{~mol}^{-1}$ and $7.710^{4} \mathrm{~cm}^{2} \mathrm{~mol}^{-1}$, respectively (39).

\section{Piezoresponse Force Microscopy switching spectroscopy}

The secondary goal of this project was to measure the piezoelectric response of the PVDF fibers. Piezoresponse Force Microscopy Switching Spectroscopy (PFM-SS) was undertaken on uniaxial fiber arrays (similar to those described previously) to monitor their vertical displacement in different voltages. Slides were cleaned using the aforementioned techniques and sputter coated with a $4 \mu \mathrm{m}$ gold layer. Fibers were then electrowritten with a potential difference of $2.8 \mathrm{kV}$, collector speed of $4800 \mathrm{~mm} / \mathrm{min}$ and $\mathrm{N}_{2}$ driving pressure of 2.0 bar. Tapping mode AFM imaging was conducted using an MFP-3D AFM (Asylum Research, CA) with a scan rate of $0.5 \mathrm{~Hz}$. A silicon tip (Pt/Ir coated) with force constant of $2.8 \mathrm{~N} / \mathrm{m}$ was used for both AFM imaging and the following PFM-SS measurements. During PFM-SS measurements, the conductive AFM tip and the gold substrate were used as top and bottom electrodes. A $200 \mathrm{mV}$ AC potential difference was applied to oscillate the tip and a sweeping DC bias (Frequency 
$=0.2 \mathrm{~Hz}$ ) in the range of $\pm 25 \mathrm{~V}$ was superimposed to induce the piezoelectric response. Within this array, PFM-SS measurement was carried on 6 different fibers, with 5 spots measured for each (30 different X-Y positions in total). The application of the sweeping DC voltage induces the polarization reversal periodically so that the piezoresponse hysteresis loop (amplitude versus $\mathrm{V}_{\mathrm{DC}}$ ) is obtained like a butterfly loop. Accordingly, the phase offers the information of the polarization on the same spot.

\section{Results and Discussion}

Based on the DSC, TG and rheology data, $190 \pm 1{ }^{\circ} \mathrm{C}$ was chosen as the processing temperature for PVDF, as it provided a good combination of polymer stability and flow characteristics as determined by preliminary rheological measurements. Visual observations showed that prolonged heat exposure caused the polymer to turn dark brown. It is assumed that within the PVDF a low molar mass additive/residual decomposes, which leads to the darkening. The melting point of PVDF was found to be at $170{ }^{\circ} \mathrm{C}$ while recrystallization occurred at $131{ }^{\circ} \mathrm{C}$ in the second heating/cooling cycle (Figure 3A). The MEW processing temperature was $20^{\circ} \mathrm{C}$ above the melting point. A stable viscosity for the molten PVDF over the entire period of experiments is essential for the various parameters investigated here to be considered valid. To check this point, an arbitrarily chosen constant shear rate of $2 \mathrm{~s}^{-1}$ was applied to the PVDF melt at $190^{\circ} \mathrm{C}$ (Figure 3B). A constant viscosity of $110 \mathrm{~Pa} \cdot \mathrm{s}$ for $5 \mathrm{~h}$ indicates that minimal thermal degradation occurs in this period, allowing the automated printing approach outlined in Figure 2 to be performed. Figure 3C shows that the PVDF melt itself displays shear-thinning behavior that is generally expected for 
polymer melts (40). Even though there was some darkening of the polymer, no thermal degradation could be detected by thermogravimetric analyses and the mass remains essentially constant at $100 \%$ (Figure 3B). In fact, instead of a mass loss, a very minor increase in the mass was observed. Since the PVDF is expected to the stable in the investigated temperature range, this very small increase in the observed mass maybe either a measurement artefact or due to oxidation of minor impurities which might correlate with the discoloration. Therefore, all data suggest sufficient stability of PVDF during MEW processing despite the observed darkening.

\section{INSERT FIGURE 3 NEAR HERE}

\section{Characterization Using ImageJ}

Linear arrays of PVDF fibers made over a series of processing conditions were electrowritten onto glass microscope slides (Figure 2). An image analysis of fiber characteristics can therefore be correlated with various processing parameters. This means when a processing parameter is altered, only a few fibers based on these conditions are made and need to be measured. To allow sufficient time for jet stabilization at a new $\mathrm{N}_{2}$ pressure parameter condition (usually 2-3 minutes), the direct writing can be performed "off-sample" for jet stabilization, before returning to deposit a set of fibers "on-sample" based on these new parameter settings - significantly increasing sample throughput.

This article is protected by copyright. All rights reserved. 
The linear fiber arrays (Figure 2D) were produced using different combinations of $\mathrm{N}_{2}$ pressure, collector speed and times as described in the methods section. The arrays were then imaged using polarization microscopy, exposure corrected, segmented and then measured through the use of ImageJ and DiameterJ in order to extract information about the diameter of the fiber triplets represented within each micrograph (Figure 2A-C). The data, in the form of a frequency distribution table, was processed using MATLAB. The fiber diameter versus collector speed was analyzed by combining the triplicate trials conducted at $\mathrm{t}=0,0.5$ and $1 \mathrm{~h}$. MATLAB was used to convert the frequency distributions into raw data. An analysis of fiber diameter versus heating time was performed by combining the results of the three trials, and using MATLAB to perform a Gaussian fit of the data in order to determine the average value and standard deviation for each pressure and collector speed at $\mathrm{t}=0,0.5,1.0,2.0,4.0,8.0$, and $10.0 \mathrm{~h}$ of continuous heating at $190{ }^{\circ} \mathrm{C}$. The results of these analyses for fiber diameter are presented in Figures 4 and 5. This reduction in the fiber diameter with increased collection speed is due to the mechanical elongation of the electrified jet (11). This reduction in fiber diameter with collector speed is in agreement with other studies involving PCL (33, 41), PHMGCL (25) and PP (22).

\section{INSERT FIGURE 4 NEAR HERE}

Figure 4 reveals trends in average fiber diameter that are consistent with previous studies on MEW, with different polymers such as PCL, polypropylene and 
poly(2-ethyl-2-oxazoline) $(21,33,42)$. For example, increasing the applied $\mathrm{N}_{2}$ pressure increases the diameter of the collected fiber (Figure 4) while increasing the collector speed reduces the diameter. There are measuring limits with this automated fiber diameter measurement; a standard deviation of $\sim 8 \mu \mathrm{m}$ was evident for most parameters, which is much greater than the standard deviation typically measured using SEM. However, this may also be attributed to the much larger sampling size and analysis bias virtually unavoidable using SEM. This variability was also generally seen to increase with slower collector speeds yet was also prominent when printing was performed at a 1.5 bar $\mathrm{N}_{2}$ pressure.

As a fiber with homogenous thickness is typically desired when performing MEW, a greater variance in diameter can serve as a method for revealing unsuitable parameter boundaries with a great level of sensitivity. Unlike the typically employed manual approaches to quantifying fiber diameter, by which the researcher may select of a subset of regions to measure (often the most visually coherent), these results are unencumbered by measurement bias and thus are a more complete representation of the PVDF scaffold sample. However, when comparing to measurements made using visual observation on the same stereomicroscope, the smaller diameters (below $\sim 25 \mu \mathrm{m}$ ) did not match the automated measurement, with the actual diameter (e.g. $\sim 17 \mu \mathrm{m})$ smaller than the automated one (e.g. $\sim 23 \mu \mathrm{m})$. As shown in previous studies for MEW, there are accuracy limits for optical microscopy for diameter measurement (43). Ideally, a more powerful form of microscope, such as an objective microscope, could be used to reduce this automated diameter measurement error for the smaller fibers. 


\section{INSERT FIGURE 5 NEAR HERE}

In general, the PVDF remained processable throughout irrespective of color and no noticeable changes in the printing characteristics were evident. Constant viscosity measurement in combination with stable fiber diameters during MEW processing for up to 10 hours (Figure 5), PVDF can be considered thermally stable enough for a working day using MEW.

\section{Birefringence}

Observing the PVDF fibers under polarized light microscopy revealed biaxial birefringence, with the fast and slow optical axis oriented along and perpendicular, to the fiber axis respectively (Figure 6B). This is evidenced by the optical extinction of the cross-polarizers during rotation (Figure 6C) and implies that there is a high-degree of anisotropic crystallinity within the PVDF fibers. The intensity of light at each angle could be quantified and plotted in a polar graph (Figure 6D).

Closer observation of fibers under polarization microscopy also revealed the presence of chevron-like segmentation along the length of the fiber (Figure 6A), with each segment displaying a slight graduated change in the birefringence axis along the segment. Interestingly, it was also seen that the direction of printing determined the direction the segments were pointing to, not unlike an arrowhead (Figure 6E). In 
contrast, SEM showed no discernible surface details corresponding to this, while using bright field optical microscopy, the junctions are very faintly visible under specific lighting conditions. To the best of our knowledge, this effect has not been demonstrated involving PVDF. It is hypothesized that this periodic birefringence may either be crystalline regions interrupted with amorphous segments, and/or periodic cracking resulting from a thermal gradient as the fiber exits the orifice of the nozzle, cools and turns almost $90^{\circ}$ on the collector. However, SEM images of the fiber surfaces (Figure 7) reveal no surface features that would be associated with such cracking.

\section{INSERT FIGURE 6 NEAR HERE}

\section{$3 D$ Printability}

Using the aforementioned techniques, the possibility of producing PVDF scaffolds with MEW is realized, with attainable architectures comparable to those of poly( $\mu$-caprolactone), the current gold standard in tissue engineering applications (11). Figures 7A-B are SEM images of a single PVDF fiber direct-written in a box shape. The upper fiber trace can be seen crossing paths, forming over the lower fiber trace indicating some viscous properties upon landing on the collector. Such fibers could be deposited quite readily with good accuracy with minimal change in fiber diameter due to fiber pulsing (11).

Moreover, multi-layered wood-pile scaffolds, where five layers have been deposited in both $\mathrm{x}$ and $\mathrm{y}$ directions could be realized (Figure 7C-D). A high quality 
morphology with a high degree of coherence within its structure as well as an accurate deposition of the fiber is obtained. However, it was observed that due to the low dielectric permittivity of PVDF, a substantial deposition of material results in an induced dielectric field strongly opposing the external field which appears to limit the scaffold thickness. This results in a repulsion, and subsequent cessation of the electrowriting processes, as a sufficiently strong electric field could no longer be maintained to initiate the electrohydrodynamic jetting of the molten polymer (44). As such, a scaffold with smaller pore sizes was more restricted in attainable height.

\section{INSERT FIGURE 7 NEAR HERE}

\section{B-phase content investigation}

During MEW, the fibers are being elongated and pulled while they are cooling down in the presence of an electric field. It can be assumed that this would have an impact on the formation of the crystalline phase in PVDF in the resulting fibers. In order to determine whether there is formation of the electroactive $\beta$-phase, the FTIR spectra for neat PVDF as well as for PVDF scaffold were obtained. The PVDF powder prior to MEW processing shows typical peak for $\alpha$-phase at $874 \mathrm{~cm}^{-1}, 762 \mathrm{~cm}^{-1}$ and 615 $\mathrm{cm}^{-1}$ (Figure 8; black line) as well as peaks attributed to the electroactive $\beta$-phase at 840 $\mathrm{cm}^{-1}$ and $1278 \mathrm{~cm}^{-1}$. The $\beta$-phase content $\mathrm{F}_{\beta}$ for the unprocessed PVDF as calculated according to equation 1 was $49 \%$, which corresponds well with values found in the literature (38). The processing of the PVDF via MEW significantly affected the amount 
of the $\beta$-phase, which was found to be $79 \%$ (Figure 8; red line) after MEW. According to the literature (37), this should be sufficient to scaffolds with adequate sensing capability. This was further confirmed using PFM technique.

\section{INSERT FIGURE 8 NEAR HERE}

\section{Piezoresponse}

Using PFM, electrowritten PVDF fibers deposited onto gold coated slides were characterized to determine their piezoelectric response. The topography images were obtained with a scan rate of $0.5 \mathrm{~Hz}$ and scan area of $2 \mu \mathrm{m} * 2 \mu \mathrm{m}$ across the surface of a single fiber. The height image and corresponding amplitude image (Figure 9A and 9B) indicate an uniform morphology of the PVDF fibers, corroborating previous analyses. The PFM-SS measurements were performed by bringing the tip into contact with the sample while applying a sweeping DC voltage. An electrical circuit is formed between the conductive AFM tip and the underlying gold layer substrate acting as top and bottom electrodes. Accordingly, the vertical displacement of the AFM tip (amplitude signal) resulting from the electrical stimulation can be attributed to the piezoelectric response of the PVDF fiber. For the PVDF fiber shown in Figure 9A, PFM-SS measurements were conducted on two individual positions (marked spot 1 and 2 (Figure 9E/F)). A summary of the different PFM-SS measurements is shown in Figure 9C.

The butterfly loops in Figure 9D are typical for piezoelectric materials with the amplitudes showing the sample expansion or contraction which confirms their 
piezoelectric response. The phase value shows a 180-degree change indicating the entire switching of PVDF dipoles has been achieved (Figure 9E). For piezoelectric materials such as PVDF, the dipoles can be aligned by the external electric field. At voltages around $\pm 20 \mathrm{~V}$, which is referred as coercive field, the amplitudes show minima values due to the direction change of the dipoles. The forward and reverse coercive fields differ from each other due to the existence of non-uniformly distributed charged defects (45). To quantitatively analyze the piezoelectric response of the microfiber, piezoelectric coefficient $\left(\mathrm{d}_{33}\right)$ are extracted from the butterfly loops and corresponding phase loops, using the following equation (45).

$$
\operatorname{Acos}(\theta)=d_{33} V_{A C} Q
$$

Where $\mathrm{A}$ is the amplitude, , is the phase, $\mathrm{V}_{\mathrm{AC}}$ is the driving voltage and $\mathrm{Q}$ is the quality factor. Specifically, $\mathrm{V}_{\mathrm{AC}}$ is $200 \mathrm{mV}$ and $\mathrm{Q}$ is 32.075 in this experiment. The $\mathrm{d}_{33}$ of each fiber is the average of the response of five spots and the error bar shows the variation. Overall, the $d_{33}$ value of the 6 fibers varies from 15.4 to $23.7 \mathrm{pm} / \mathrm{V}$ (Figure 9C). Liu et al. reported a $d_{33}$ value from $38.5 \mathrm{pm} / \mathrm{V}$ to $17.6 \mathrm{pm} / \mathrm{V}$ for electrospun PVDF nanofibers with diameters in between $30 \mathrm{~nm}$ to $300 \mathrm{~nm}(45,46)$. The comparable but somewhat lower piezoresponse obtained from the PVDF microfibers may be attributed to the larger diameters fabricated by MEW. It is also reported that the $\mathrm{d}_{33}$ value changes along both the diameter direction and the longitudinal direction, (47), which explains the different piezoresponse obtained from different PVDF fibers and the error bars. However, the data unambiguously show that the MEW-processed PVDF fibers show electroactive behavior and thus, should be suitable as sensor or actuator material. 
Compared to electrospun or spin-cast PVDF, MEW offers a unique potential for anisotropic designer sensors/actuators.

\section{INSERT FIGURE 9 NEAR HERE}

\section{Conclusion}

In conclusion, there are numerous advances described in this study. We have shown for the first time that MEW of PVDF is possible and the fiber deposition can be well-controlled, enabling the fabrication of complex, 3-dimensional structures. Moreover, the direct-written PVDF fibers exhibit sufficient transformation of the electroactive crystalline phase which reaches approx. 79\%. The direct-written fibers attain a piezoresponse showing $19 \mathrm{pm} / \mathrm{V}$ which is of sufficient magnitude for use in sensing applications. Moreover, a method for the rapid analysis and characterization of MEW scaffolds was also introduced, allowing for an accurate approach to the quantification of fiber diameter while many different instrument parameters are changed. Our results provide a solid platform upon which further studies and applications of PVDF fibers can be explored.

\section{Acknowledgements}

The assistance of Mr. Andrei Hrynevich for SEM imaging is appreciated. The EACEA program BIOFAB (grant\# 2013/3137 001-001) to supported SF, while the Volkswagen Stiftung (Grant\# 93 417) is greatly appreciated for their financial assistance. The German Research Foundation (DFG) State Major Instrumentation 
Programme (INST 105022/58-1 FUGG) funded the Zeiss Crossbeam CB 340 SEM used in this study. We acknowledge the Australian Research Council (ARC) Centre of Excellence for Electromaterials Science (CE 140100012) for financial support and the Australian National Fabrication Facility (ANFF)-Materials Node and University of Wollongong for use of facilities. The support of ARC Industrial Transformation Training Centre in Additive Biomanufacturing (IC160100026) is also acknowledged.

This article is protected by copyright. All rights reserved. 


\section{References}

1. Melchels FPW, Domingos MAN, Klein TJ, Malda J, Bartolo PJ, Hutmacher DW. Additive manufacturing of tissues and organs. Prog Polym Sci. 2012;37(8):1079104.

2. Zein I, Hutmacher DW, Tan KC, Teoh SH. Fused deposition modeling of novel scaffold architectures for tissue engineering applications. Biomaterials. 2002;23(4):1169-85.

3. Jungst T, Smolan W, Schacht K, Scheibel T, Groll J. Strategies and molecular design criteria for 3D printable hydrogels. Chem Rev. 2016;116(3):1496-539.

4. Lorson T, Jaksch S, Lubtow MM, Jungst T, Groll J, Luhmann T, et al. A Thermogelling Supramolecular Hydrogel with Sponge-Like Morphology as a Cytocompatible Bioink. Biomacromolecules. 2017;18(7):2161-71.

5. Smay JE, Gratson GM, Shepherd RF, Cesarano J, Lewis JA. Directed colloidal assembly of 3D periodic structures. Adv Mater. 2002;14(18):1279-83.

6. Kishi N, Iizuka H. Barus effect of polymer melts. J Polym Sci Part B-Polym Lett. 1964;2:399-402.

7. Dalton PD. Melt electrowriting with additive manufacturing principles. Current Opinion in Biomedical Engineering. 2017;2:49-57.

8. Huang Y, Bu N, Duan Y, Pan Y, Liu H, Yin Z, et al. Electrohydrodynamic direct-writing. Nanoscale. 2013;5(24):12007-17.

9. Reneker DH, Chun I. Nanometre diameter fibres of polymer, produced by electrospinning. Nanotechnology. 1996;7(3):216-23.

10. Taylor G. Electrically Driven Jets. Proceedings of the Royal Society of London. 1969;313:453-75.

11. Hochleitner G, Youssef A, Hrynevich A, Haigh JN, Jungst T, Groll J, et al. Fibre pulsing during melt electrospinning writing. BioNanoMaterials. 2016;17(3):159-71.

12. Hochleitner G, Chen F, Blum C, Dalton PD, Amsden B, Groll J. Melt electrowriting below the critical translation speed to fabricate crimped elastomer scaffolds with non-linear extension behaviour mimicking that of ligaments and tendons. Acta Biomater. 2018;72:110-20.

13. Greiner A, Wendorff JH. Electrospinning: A fascinating method for the preparation of ultrathin fibres. Angewandte Chemie-International Edition. 2007;46(30):5670-703.

14. Larrondo L, Manley RSJ. Electrostatic fiber spinning from polymer melts.1. Experimental observations on fiber formation and properties. Journal of Polymer Science Part B-Polymer Physics. 1981;19(6):909-20.

15. Brown TD, Dalton PD, Hutmacher DW. Melt electrospinning today: An opportune time for an emerging polymer process. Prog Polym Sci. 2016;56:116-66.

16. Fang J, Zhang L, Sutton D, Wang X, Lin T. Needleless melt-electrospinning of polypropylene nanofibres. Journal of Nanomaterials. 2012;2012:1-9.

17. Melgoza EL, Vallicrosa G, Sereno L, Ciurana J, Rodriguez CA. Rapid tooling using 3D printing system for manufacturing of customized tracheal stent. Rapid Prototyping J. 2014;20(1):2-12. 
18. Visser J, Melchels FPW, Jeon JE, van Bussel EM, Kimpton LS, Byrne HM, et al. Reinforcement of hydrogels using three-dimensionally printed microfibres. Nature communications. 2015;6:DOI:10.1038/ncomms7933.

19. de Ruijter M, Hrynevich A, Haigh JN, Hochleitner G, Castilho M, Groll J, et al. Out-of-Plane 3D-Printed Microfibers Improve the Shear Properties of Hydrogel Composites. Small. 2018;14(8).

20. Brown TD, Dalton PD, Hutmacher DW. Direct writing by way of melt electrospinning. Adv Mater. 2011;23(47):5651-7.

21. Hochleitner G, Hummer JF, Luxenhofer R, Groll J. High definition fibrous poly(2-ethyl-2-oxazoline) scaffolds through melt electrospinning writing. Polymer. 2014;55(20):5017-23.

22. Haigh JN, Dargaville TR, Dalton PD. Additive manufacturing with polypropylene microfibers. Materials Science and Engineering: C. 2017;77:883-7.

23. Chen F, Hochleitner G, Woodfield T, Groll J, Dalton PD, Amsden BG. Additive manufacturing of a photo-cross-linkable polymer via direct melt electrospinning writing for producing high strength structures. Biomacromolecules. 2016;17(1):208-14.

24. Hochleitner G, Fürsattel E, Giesa R, Groll J, Schmidt HW, Dalton PD. Melt Electrowriting of Thermoplastic Elastomers. Macromolecular Rapid Communications. 2018;0(0):1800055.

25. Castilho M, Feyen D, Flandes-Iparraguirre M, Hochleitner G, Groll J, Doevendans PAF, et al. Melt Electrospinning Writing of PolyHydroxymethylglycolide-co-epsilon-Caprolactone-Based Scaffolds for Cardiac Tissue Engineering. Adv Healthc Mater. 2017;6(18).

26. A. IA, Mariam AM, S. LA, Miroslav M, K. HM. Investigation of the physico mechanical properties of electrospun PVDF/cellulose (nano)fibers. Journal of Applied Polymer Science. 2016;133(26).

27. Zeng W, Tao X-M, Chen S, Shang S, Chan HLW, Choy SH. Highly durable allfiber nanogenerator for mechanical energy harvesting. Energ Environ Sci. 2013;6(9):2631-8.

28. Jia N, Xing Q, Xia G, Sun J, Song R, Huang W. Enhanced ${ }^{2}$-crystalline phase in poly(vinylidene fluoride) films by polydopamine-coated $\mathrm{BaTiO} 3$ nanoparticles. Materials Letters. 2015;139:212-5.

29. Fang J, Niu H, Wang H, Wang X, Lin T. Enhanced mechanical energy harvesting using needleless electrospun poly(vinylidene fluoride) nanofibre webs. Energ Environ Sci. 2013;6(7):2196-202.

30. Sharma M, Madras G, Bose S. Process induced electroactive ${ }^{2}$-polymorph in PVDF: effect on dielectric and ferroelectric properties. Physical Chemistry Chemical Physics. 2014;16(28):14792-9.

31. He FA, Sarkar M, Lau S, Fan JT, Chan LH. Preparation and characterization of porous poly(vinylidene fluoride-trifluoroethylene) copolymer membranes via electrospinning and further hot pressing. Polymer Testing. 2011;30(4):436-41.

32. Hochleitner G, Jungst T, Brown TD, Hahn K, Moseke C, Jakob F, et al. Additive manufacturing of scaffolds with sub-micron filaments via melt electrospinning writing. Biofabrication. 2015;7(3):035002. 
33. Hrynevich A, Elci BS, Haigh JN, McMaster R, Youssef A, Blum C, et al. Dimension-Based Design of Melt Electrowritten Scaffolds. Small. 2018;14(22):e1800232.

34. Hotaling NA, Bharti K, Kriel H, Simon CG. DiameterJ: A validated open source nanofiber diameter measurement tool. Biomaterials. 2015;61:327-38.

35. Narayanan G, Tekbudak MY, Caydamli Y, Dong J, Krause WE. Accuracy of electrospun fiber diameters: The importance of sampling and person-to-person variation. Polymer Testing. 2017;61:240-8.

36. Allen ACB, Barone E, Crosby CO, Suggs LJ, Zoldan J. Electrospun poly(Nisopropyl acrylamide)/poly(caprolactone) fibers for the generation of anisotropic cell sheets. Biomater Sci-Uk. 2017;5(8):1661-9.

37. Ting Y, Suprapto, Nugraha A, Chiu CW, Gunawan H. Design and characterization of one-layer PVDF thin film for a 3D force sensor. Sensors and Actuators A: Physical. 2016;250:129-37.

38. Salimi A, Yousefi AA. Analysis Method: FTIR studies of ${ }^{2}$-phase crystal formation in stretched PVDF films. Polymer Testing. 2003;22(6):699-704.

39. Gomes J, Nunes JS, Sencadas V, Lanceros-Mendez S. Influence of the ${ }^{2}$-phase content and degree of crystallinity on the piezo- and ferroelectric properties of poly(vinylidene fluoride). Smart Mater Struct. 2010;19(6):065010.

40. Kalambur S, Rizvi SSH. Rheological behavior of starch-polycaprolactone (PCL) nanocomposite melts synthesized by reactive extrusion. Polymer Engineering and Science. 2006;46(5):650-8.

41. Bas O, Lucarotti S, Angella DD, Castro NJ, Meinert C, Wunner FM, et al. Rational design and fabrication of multiphasic soft network composites for tissue engineering articular cartilage: A numerical model-based approach. Chem Eng J. 2018;340:15-23.

42. Haigh JN, Dargaville TR, Dalton PD. Additive Manufacturing of Polypropylene. Materials science \& engineering C, Materials for biological applications. submitted.

43. Brown TD, Edin F, Detta N, Skelton AD, Hutmacher DW, Dalton PD. Melt electrospinning of poly(epsilon-caprolactone) scaffolds: Phenomenological observations associated with collection and direct writing. Materials Science \& Engineering CMaterials for Biological Applications. 2014;45:698-708.

44. Wunner FM, Florczak S, Mieszczanek P, Bas O, De-Juan-Pardo EM, Hutmacher DW. Electrospinning With Polymer Melts - State of the Art and Future Perspectives. In: Ducheyne P, editor. Comprehensive Biomaterials II. Oxford: Elsevier; 2017. p. 21735.

45. Liu $\mathrm{X}$, Kuang $\mathrm{X}, \mathrm{Xu} \mathrm{S}$, Wang $\mathrm{X}$. High-sensitivity piezoresponse force microscopy studies of single polyvinylidene fluoride nanofibers. Materials Letters. 2017;191:189-92.

46. Tian Z, Zhilian Y, Gordon GW, Yi D, Pedro M, Senentxu L-M, et al. Local probing of magnetoelectric properties of PVDF/Fe $3 \mathrm{O} 4$ electrospun nanofibers by piezoresponse force microscopy. Nanotechnology. 2017;28(6):065707. 
47. Liu X, Deng M, Wang X. Nanoscale domain imaging and local piezoelectric coefficient d33 studies of single piezoelectric polymeric nanofibers. Materials Letters. 2017; 189:66-9.

This article is protected by copyright. All rights reserved. 


\section{Figure Legends}

Figure 1. Schematic of the MEW jet and fibers, with some of the essential parameters that affect fiber diameter/quality labelled. The variables measured in this study are shown in yellow.

Figure 2. Demonstration of grayscale JPEG image conversion (microscope image, A) of electrowritten fibers to a black and white binary segmented image (B) ready for analysis. C) Thresholding algorithms dictate how the background and foreground are isolated. Microscope images originate from D) the printing array of the fibers for each time period up to $10 \mathrm{~h}$. Two slides are used for one printing run to accommodate a pressure range from 1.5 bar to 3.0 bar (as shown in red). A jet stabilization routine (blue) is performed when transitioning to another $\mathrm{N}_{2}$ pressure. Each fiber triplet corresponds to a collector speed from $1800 \mathrm{~mm} / \mathrm{min}$ to $5000 \mathrm{~mm} / \mathrm{min}$ (green) and are the source of the images for conversion.

Figure 3. PVDF analysis displaying A) the DSC thermogram showing the melting transition and recrystallization, B) the viscosity and gravimetric loss at $190^{\circ} \mathrm{C}$ for $5 \mathrm{~h}$ (rotational shear rate of ${ }^{3}=2 \mathrm{~s}^{-1}$ ) and C) the shear rate dependent viscosity

Figure 4. Fiber diameter versus collector speed and $\mathrm{N}_{2}$ driving pressure. Each point is a culmination of nine trials consisting of three scaffolds printed at 30 minute intervals, in triplicate over three different days.

Figure 5. Graph of the fiber diameter versus time as obtained from Gaussian fits of the diameter frequency distributions. Here the data is grouped at different $\mathrm{N}_{2}$ pressures. 
Figure 6. A) Photograph of a PVDF scaffold made by MEW, placed under a $1 \mathrm{~mm}$ thick microscope slide to ensure entire structure remained within the plane of focus. B) SEM micrograph of uncoated electrowritten PVDF fiber. C) Image of same fiber under polarization microscopy with visible segmentation. D) Optical extinction of fiber when oriented at different angles. E) Radial plot of normalized grayscale intensity as a function of polarizer angle. F) Image highlighting the influence of electrowriting direction on the structure of the segmentation.

Figure 7. SEM images of A) single-a square lay-down pattern of MEW fibres and B) magnification of the PVDF fibre surface. C) A 5-layer PVDF scaffold with a pore size of $500 \mu \mathrm{m}$ is shown while D) the enlarged magnification shows the slight thermal fusion (white arrow) that occurs at the intersection points.

Figure 8. FTIR spectra of the neat PVDF (black line) and MEW-processed PVDF (red line).

Figure 9. A) Height image and B) corresponding amplitude image are given, with C) showing the outcomes of PFM Switching Spectroscopy (PFM-SS). Conducted on 30 different X-Y positions ( $\left.5^{*} 6\right)$, only two spots are shown here, with D and E correlating with the two spots in A).

This article is protected by copyright. All rights reserved. 


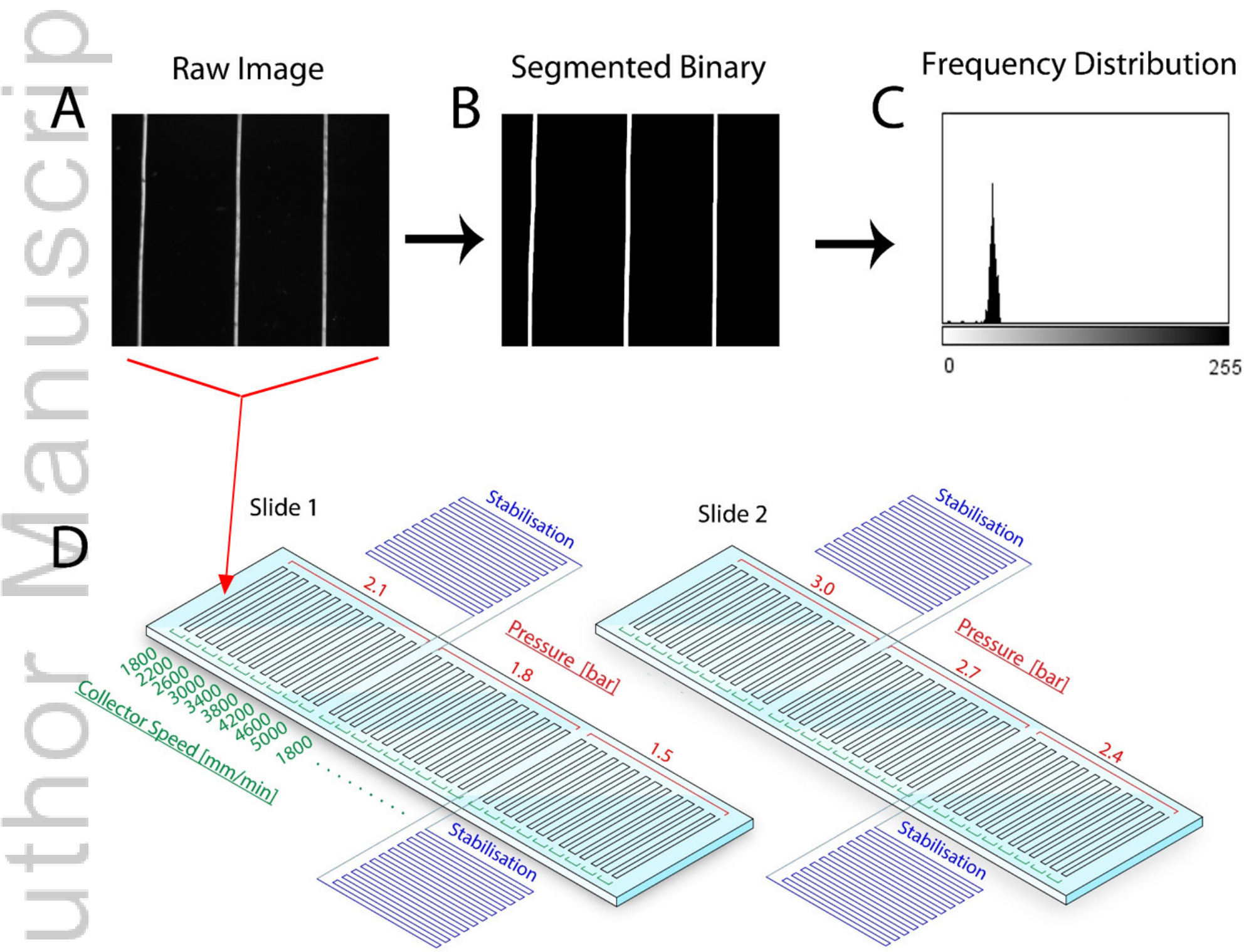

Figure 2 Final.jpg 

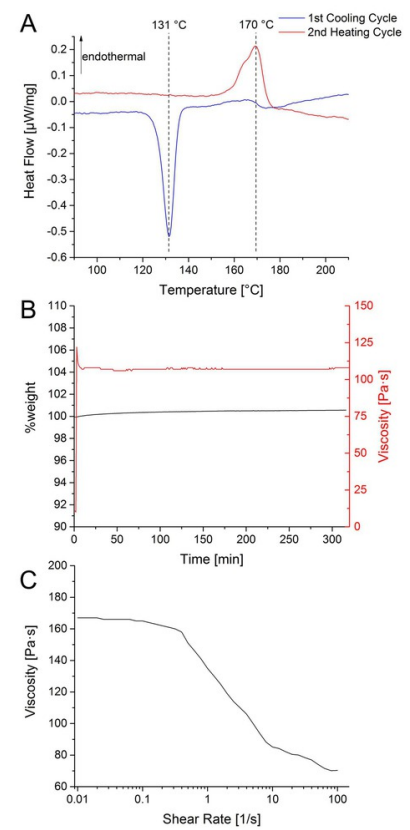

Figure 3 New Final Updated.jpg

This article is protected by copyright. All rights reserved. 

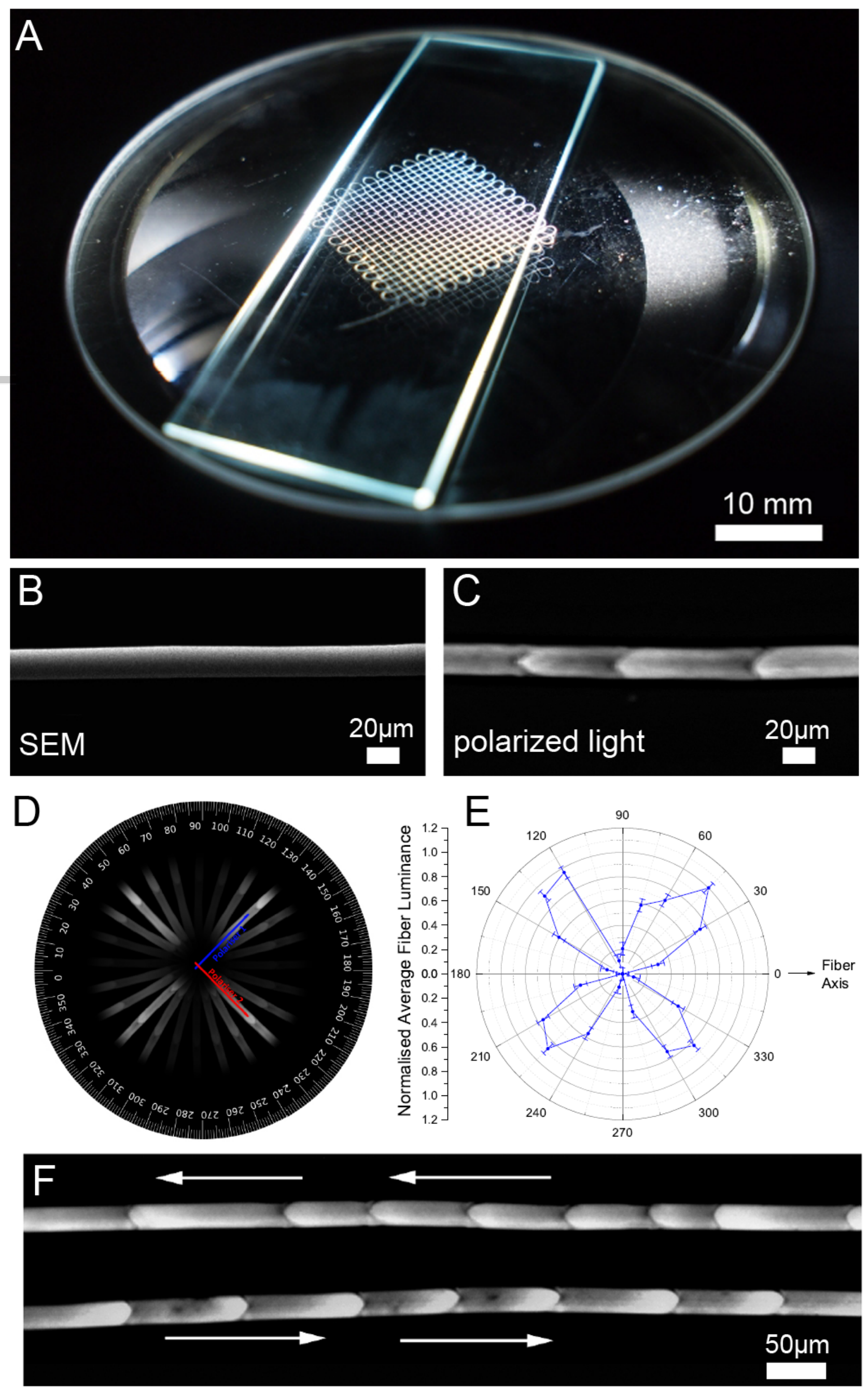

Figure 6 Final.tif

This article is protected by copyright. All rights reserved. 

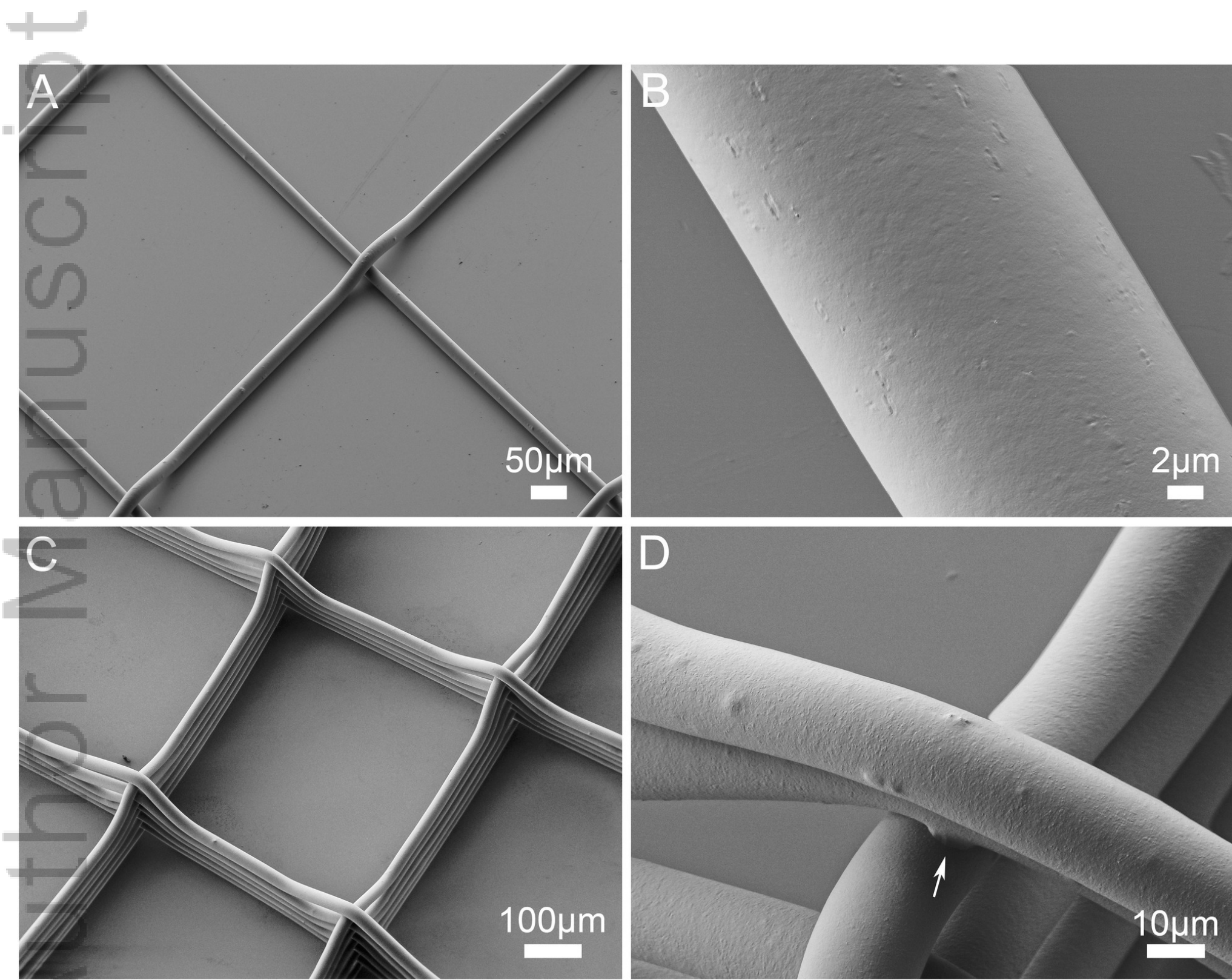

Figure 7 Final.jpg

This article is protected by copyright. All rights reserved. 

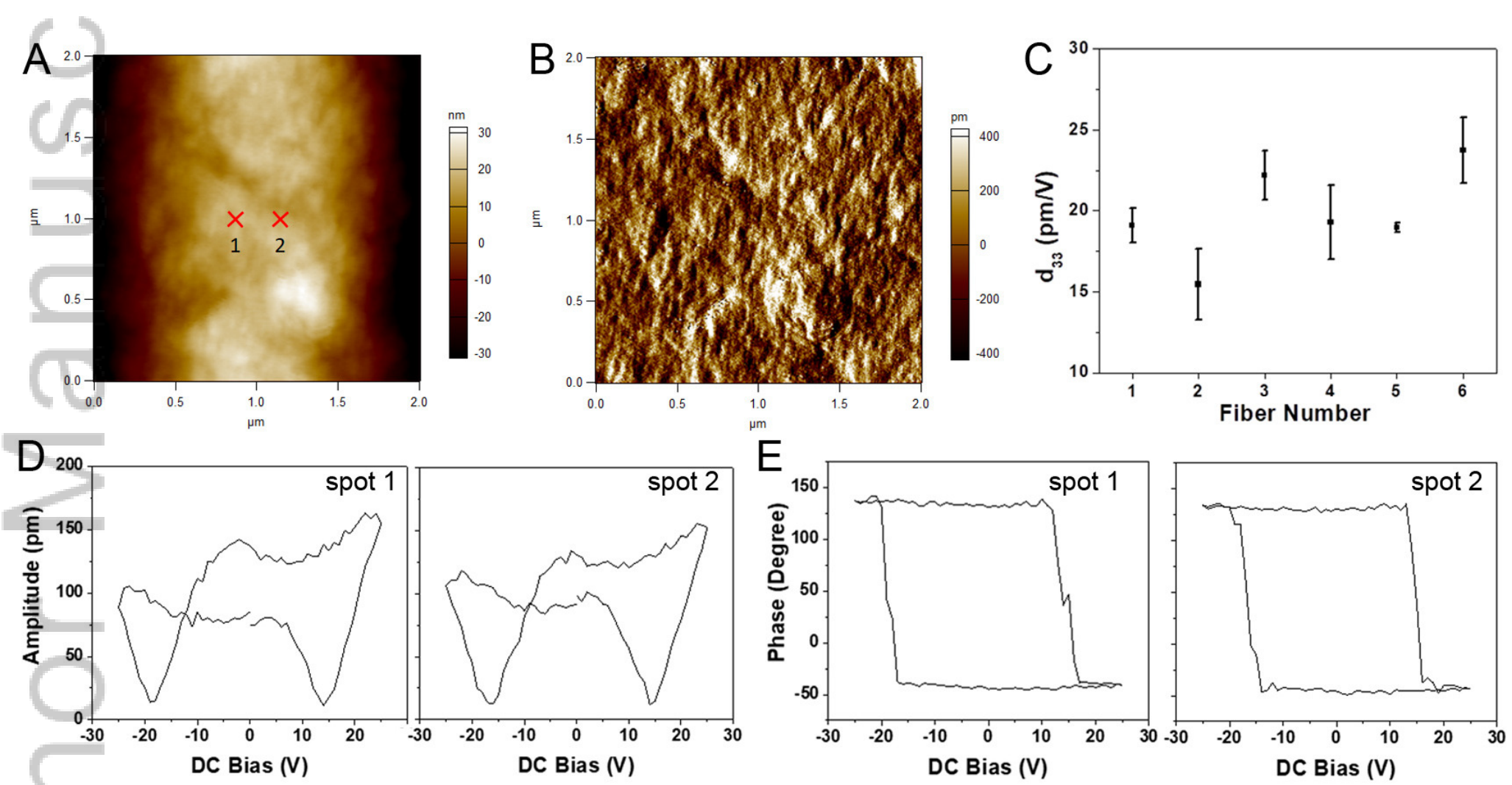

Figure 9 Final.jpg

This article is protected by copyright. All rights reserved. 


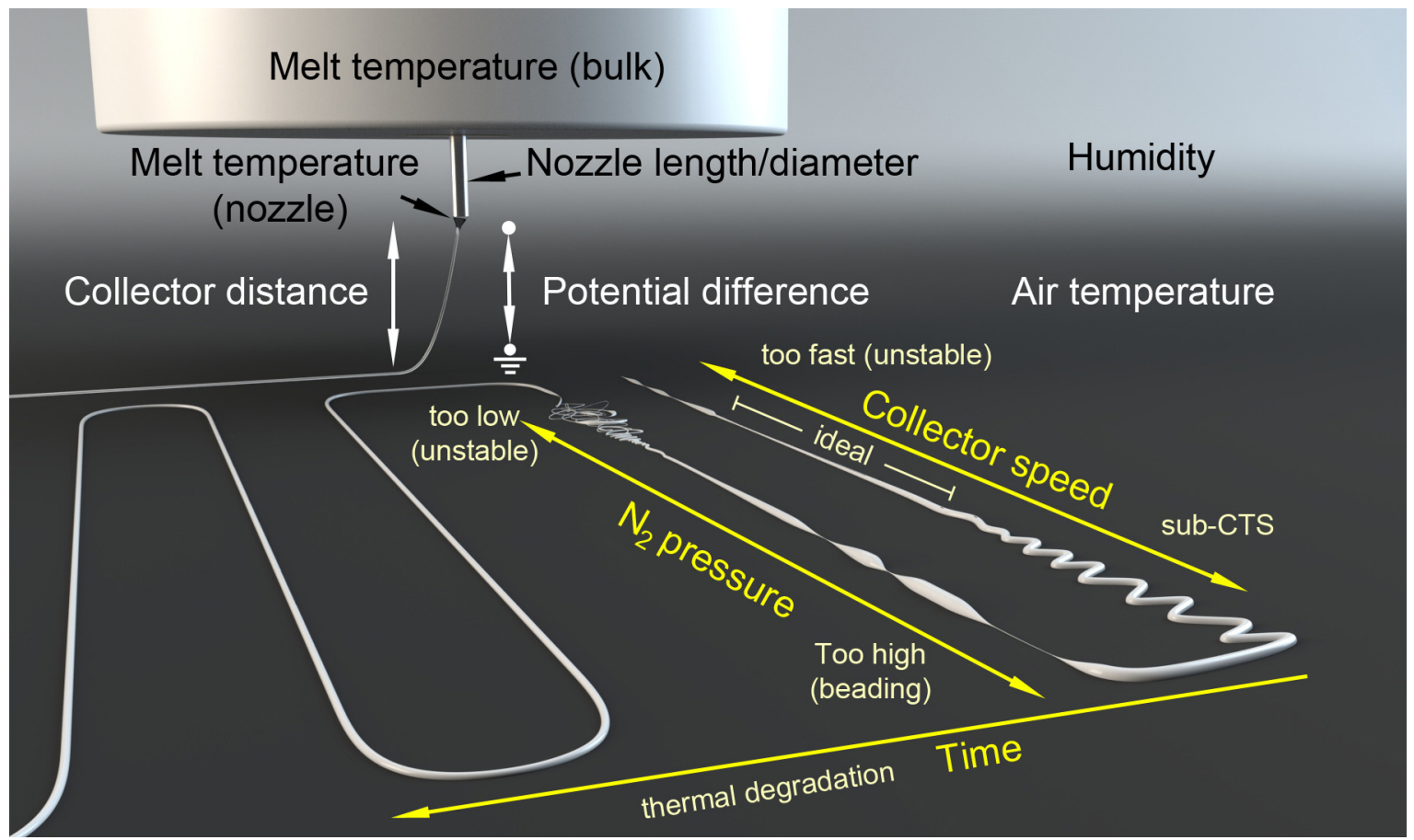

FIgure 1 PVDF New V2.jpg

This article is protected by copyright. All rights reserved. 

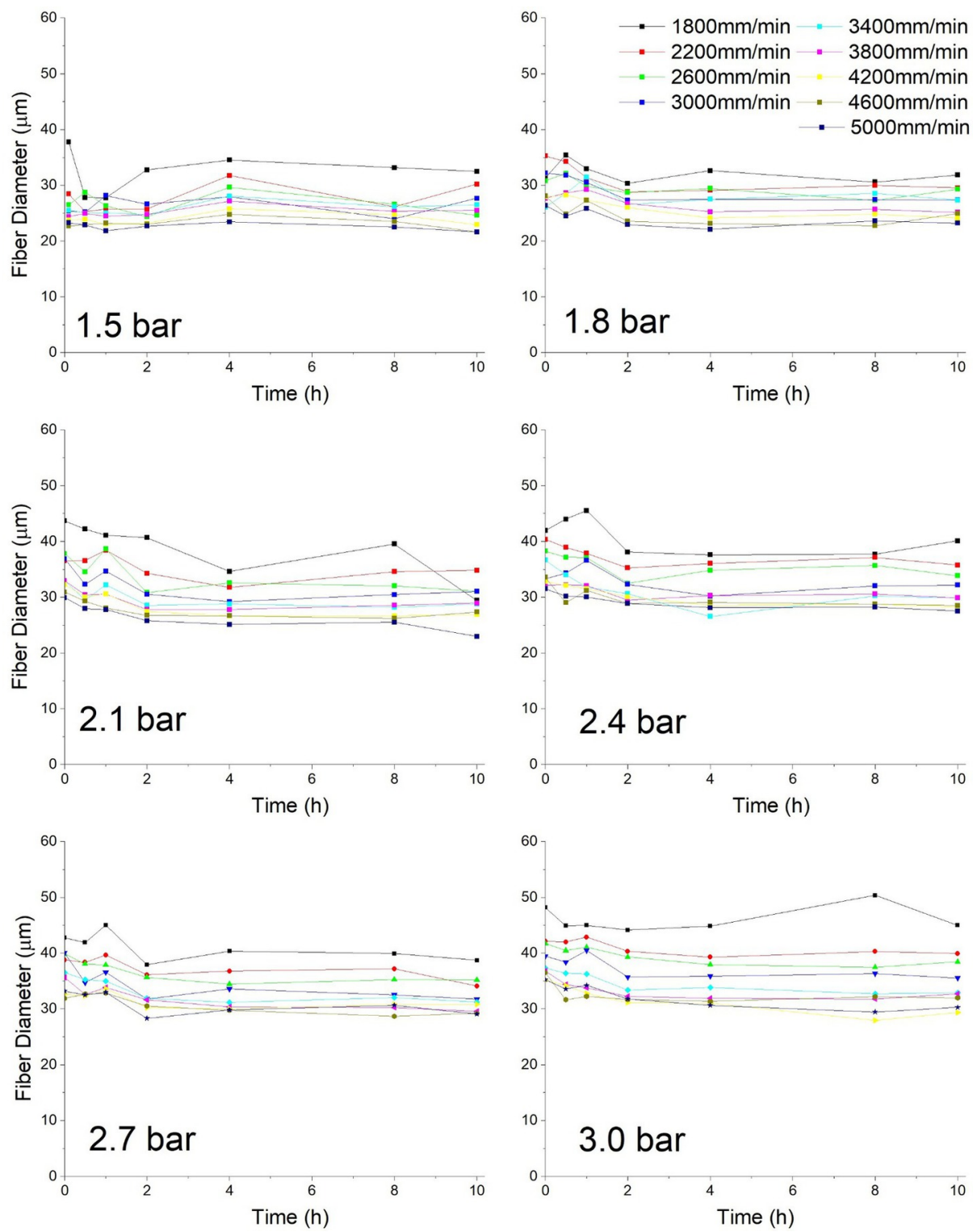

FIgure 5 FInal.jpg

This article is protected by copyright. All rights reserved. 


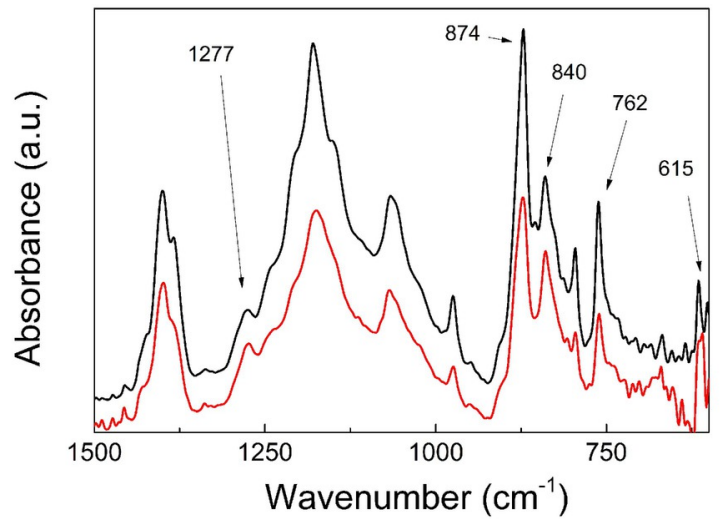

FIgure 8 FInal.jpg

This article is protected by copyright. All rights reserved. 
Melt Electrowriting of Electroactive Poly(vinylidene difluoride) Fibers

Sammy Florczak, Thomas Lorson, Tian Zheng, Miroslav Mrlik,

Dietmar W. Hutmacher, Michael J. Higgins, Robert Luxenhofer*, Paul D. Dalton*

Poly(vinylidene difluoride) was 3D-printed via melt electrowriting into small diameter fibers that were electroactive without poling.

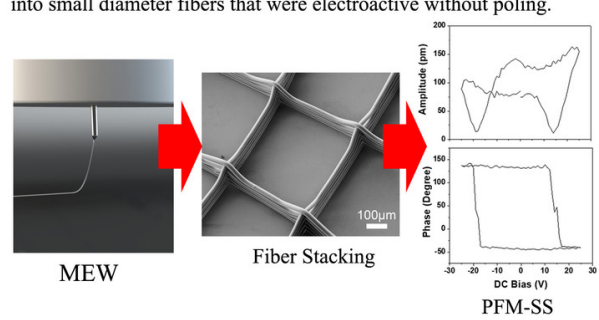

Graphical Abstract.jpg

This article is protected by copyright. All rights reserved. 


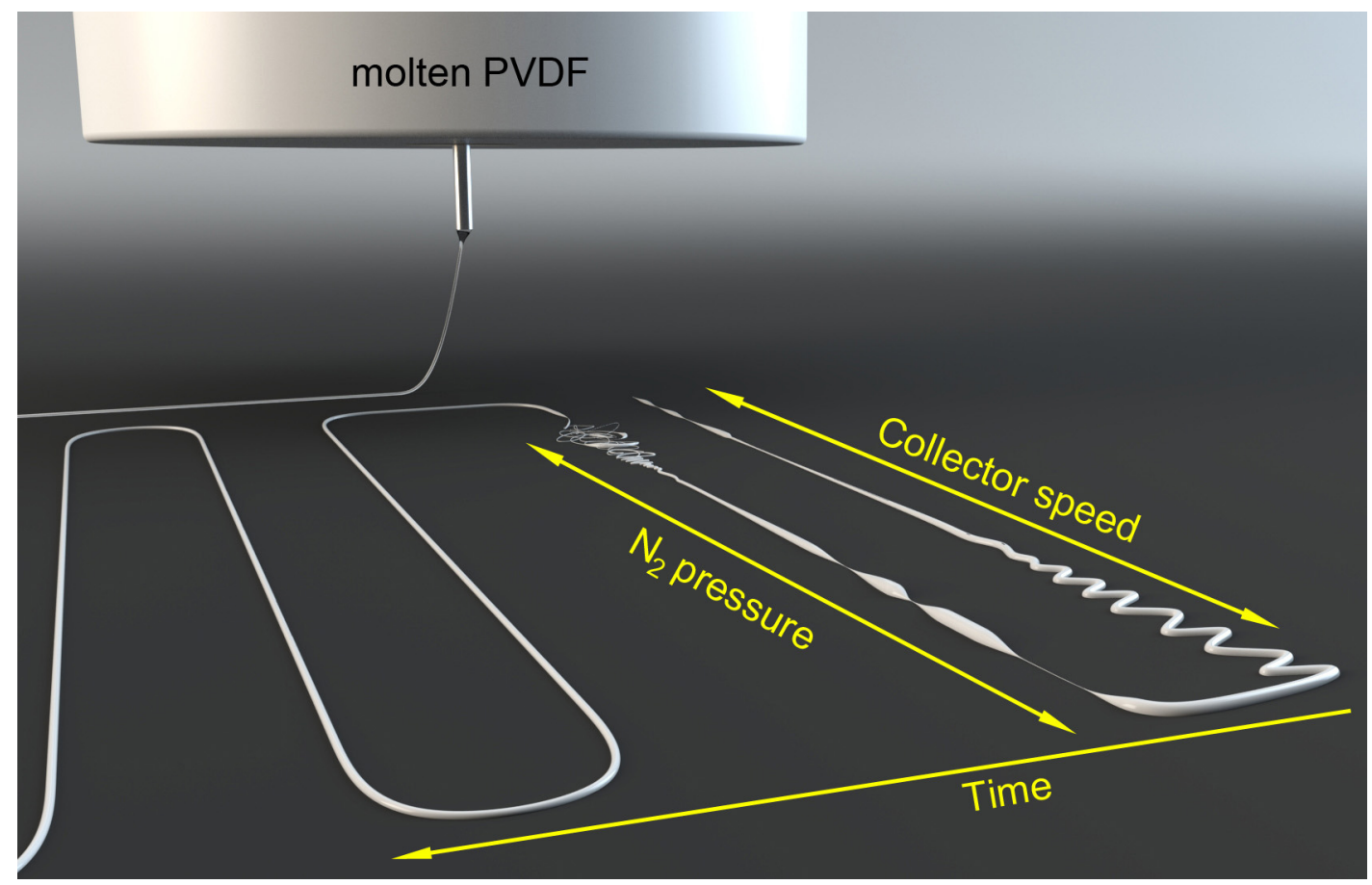

Graphical Figure.jpg

This article is protected by copyright. All rights reserved. 


\section{University Library}

\section{- M M I N E R VA A gateway to Melbourne's research publications}

Minerva Access is the Institutional Repository of The University of Melbourne

\section{Author/s:}

Florczak, S;Lorson, T;Zheng, T;Mrlik, M;Hutmacher, DW;Higgins, MJ;Luxenhofer, R;Dalton, PD

Title:

Melt electrowriting of electroactive poly(vinylidene difluoride) fibers

Date:

2019-04-01

\section{Citation:}

Florczak, S., Lorson, T., Zheng, T., Mrlik, M., Hutmacher, D. W., Higgins, M. J., Luxenhofer, R. \& Dalton, P. D. (2019). Melt electrowriting of electroactive poly(vinylidene difluoride) fibers. Polymer International, 68 (4), pp.735-745. https://doi.org/10.1002/pi.5759.

Persistent Link:

http://hdl.handle.net/11343/285358 